\title{
Successful treatment of BK virus associated-nephropathy in a human immunodeficiency virus-positive kidney transplant recipient
}

International Journal of STD \& AIDS 0 (0) $1-5$

(C) The Author(s) 2020 Article reuse guidelines: sagepub.com/journals-permissions DOI: $10.1177 / 0956462419900853$ journals.sagepub.com/home/std @SAGE

\author{
Gaetano Alfano ${ }^{1,2}$ (D), Francesco Fontana', Giovanni Guaraldi ${ }^{3}$, \\ Gianni Cappelli, ${ }^{1,2}$ and Cristina Mussini ${ }^{3}$
}

\begin{abstract}
$B K$ virus $(B K V)$ is an opportunistic pathogen in those with impaired immunity. Viral replication is generally asymptomatic but is able to induce cytopathic alterations in renal cells. If BKV infection is left untreated, it leads to BKV-associated nephropathy (BKVAN) and graft loss. There is scarce experience in the management of BKV infection in kidney transplant recipients living with HIV. We report the successful treatment of BKVAN in an HIV-positive kidney transplant recipient who experienced $\mathrm{BKV}$ replication in the immediate post-transplantation period. A change in therapy from calcineurin inhibitor to sirolimus, steroid withdrawal and a short course of an immunomodulatory agent (leflunomide) controlled BKV viremia in the absence of drug side-effects or impairment of graft function.
\end{abstract}

\section{Keywords}

HIV, BK virus, sirolimus, mTORi, antiretroviral therapy, kidney transplantation, rapamycin

Date received: 19 October 2019; accepted: 16 December 2019

\section{Introduction}

Kidney transplantation is a safe and effective option for end-stage renal disease (ESRD) in human immunodeficiency virus (HIV)-positive kidney transplant (KT) recipients. ${ }^{1-4}$ It has been associated with a better outcome compared to dialysis treatment. However, the use of antirejection agents exposes this vulnerable population to multiple opportunistic infections. BK virus (BKV) is a pathogen frequently encountered after kidney transplantation. The manifestations are heterogeneous and vary from a sub-clinical viruria to BKV-associated nephropathy (BKVAN). Treatment of BKV infection remains undetermined and consists primarily of the reduction of immunosuppression. ${ }^{5}$ This strategy has been offset by a higher risk of graft rejections. ${ }^{6,7}$ In the last decade, sirolimus (SIR) and everolimus, two immunosuppressive agents with multiple pleiotropic activities, have been shown to be effective against BKV in few retrospective ${ }^{8-11}$ and prospective studies ${ }^{12,13}$ limited to kidney transplantation. Given the scarce experience about the management of
BKV infection in people living with HIV, we report our therapeutic strategy in controlling sustained BKV replication in an HIV-positive KT recipient.

\section{Case presentation}

A 45-year-old Caucasian female with a diagnosis of HIV-1 infection from 1995 progressed to end-stage renal disease in March 2014. Renal failure was secondary to repeated episodes of acute pyelonephritis. She

\footnotetext{
'Nephrology Dialysis and Transplant Unit, University Hospital of Modena, Modena, Italy

${ }^{2}$ Surgical, Medical and Dental Department of Morphological Sciences, Section of Nephrology, University of Modena and Reggio Emilia, Modena, Italy

${ }^{3}$ Clinic of Infectious Diseases, University of Modena and Reggio Emilia, University Hospital of Modena, Modena, Italy
}

\section{Corresponding author:}

Gaetano Alfano, University Hospital of Modena, Via del Pozzo, 7I, 4I I 24, Modena, Italy.

Email: gaetano.alfano@unimore.it 


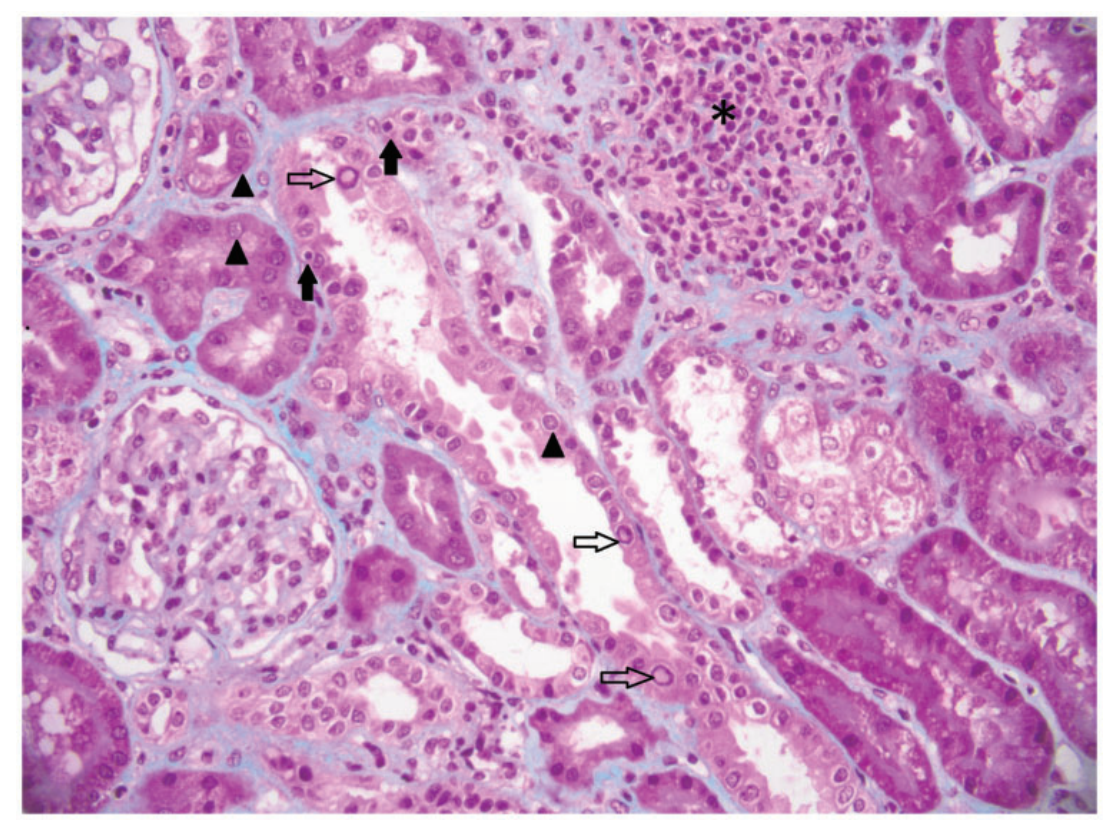

Figure I. Masson's trichrome stain reveals regional interstitial inflammation with prevalence of mononuclear cells $\left(^{*}\right)$. Tubular injury is characterized by tubular desquamation, flattened epithelial lining and mild tubulitis (full arrow). Cytopathic effects of polyomavirus infection are seen prevalently in tubular cells. Nuclei of infected tubular cells are diffusely enlarged and often have a surrounding halo. Some nuclei are characterized by a central irregular inclusion body surrounded by a halo (empty arrow), others by finely granular alterations (arrowhead).

had never experienced an AIDS-defining illness and the nadir of her CD4+ T-cell count was 386 cells $/ \mathrm{mm}^{3}$ in February 1998. Antiretroviral therapy (ART) was started soon after this drop in CD4 cell count.

After one year of peritoneal dialysis, she received a deceased donor KT in March 2015. Before transplantation, her HIV viral load (VL) was undetectable and CD4+ T-cell count was 1146 cells $/ \mathrm{mm}^{3}$. Her ART consisted of lamivudine/raltegravir/rilpivirine (3TC/RAL/ RPV). The patient received basiliximab and steroid as induction therapy and tacrolimus (TAC) and prednisone as maintenance therapy. She had immediate allograft function and was discharged with a creatinine of $1 \mathrm{mg} / \mathrm{dl}\left(\mathrm{eGFR},{ }^{14} 63 \mathrm{ml} / \mathrm{min}\right)$ on postoperative day 14. After transplantation, ART was changed to raltegravir/maraviroc/rilpivirine (RAL/ MVC/RPV). Valganciclovir and co-trimoxazole were used as prophylaxis for cytomegalovirus and pneumocystis infections, respectively.

Mean TAC trough level was $10.1 \mathrm{mg} / \mathrm{dl}$ during the first two months. At the end of the first month after transplantation, measurement of BK viral load by realtime polymerase chain reaction (BKV ELITe $\mathrm{MGB}^{\circledR}$ Kit) revealed a viruria and viremia of $5.4 \log 10$ and $2.5 \log 10$ copies/mL, respectively. In December 2015, a progressive and concerning increase of viruria (8.4 $\log 10$ copies $/ \mathrm{mL})$ and viremia $(3.8 \log 10$ copies $/ \mathrm{mL})$ prompted early steroid withdrawal in order to enhance host immunocompetence against BKV. In March 2016, graft biopsy was performed for a progressive decline in renal function (serum creatinine 1.27 vs. $1 \mathrm{mg} / \mathrm{dl}$ ). It revealed viral cytogenic alterations suggestive of BKVAN. Microscopic evaluation showed regional interstitial inflammation with a prevalence of mononucleated cells and several tubular epithelial cells with intranuclear viral inclusion bodies typical of viral infection (Figure 1). In some tubules, the proliferation of BKV manifested as tubulitis. SV40 T-antigen antibody provided negative results. Lack of endarteritis, negative staining for $\mathrm{C} 4 \mathrm{~d}$ on graft biopsy and absence of blood donor-specific antibodies (DSA) excluded the diagnosis of graft rejection. No signs of calcineurin toxicity were found in the biopsy specimen.

Based on these findings, TAC was switched to SIR for its antiproliferative proprieties in April 2016. SIR trough level was maintained between 5 and $8 \mathrm{ng} / \mathrm{dl}$. In order to favor the resolution of BKV, she started leflunomide at a standard dose of $40 \mathrm{mg}$ daily, but the drug was prematurely discontinued due to severe pancytopenia. After an early BK peak viruria of $10 \log 10$ copies $/ \mathrm{mL}$ and viremia of $6.3 \log 10$ copies $/ \mathrm{mL}$, serum viral load declined progressively to about $2.0 \log 10$ in 2017 and remained constantly stable until July 2018 despite a normal CD4+ T-cell count (Figure 2).

At the end of the follow-up, her creatinine level settled to $1.5 \mathrm{mg} / \mathrm{dl}$ corresponding to an $\mathrm{eGFR}^{14}$ of 


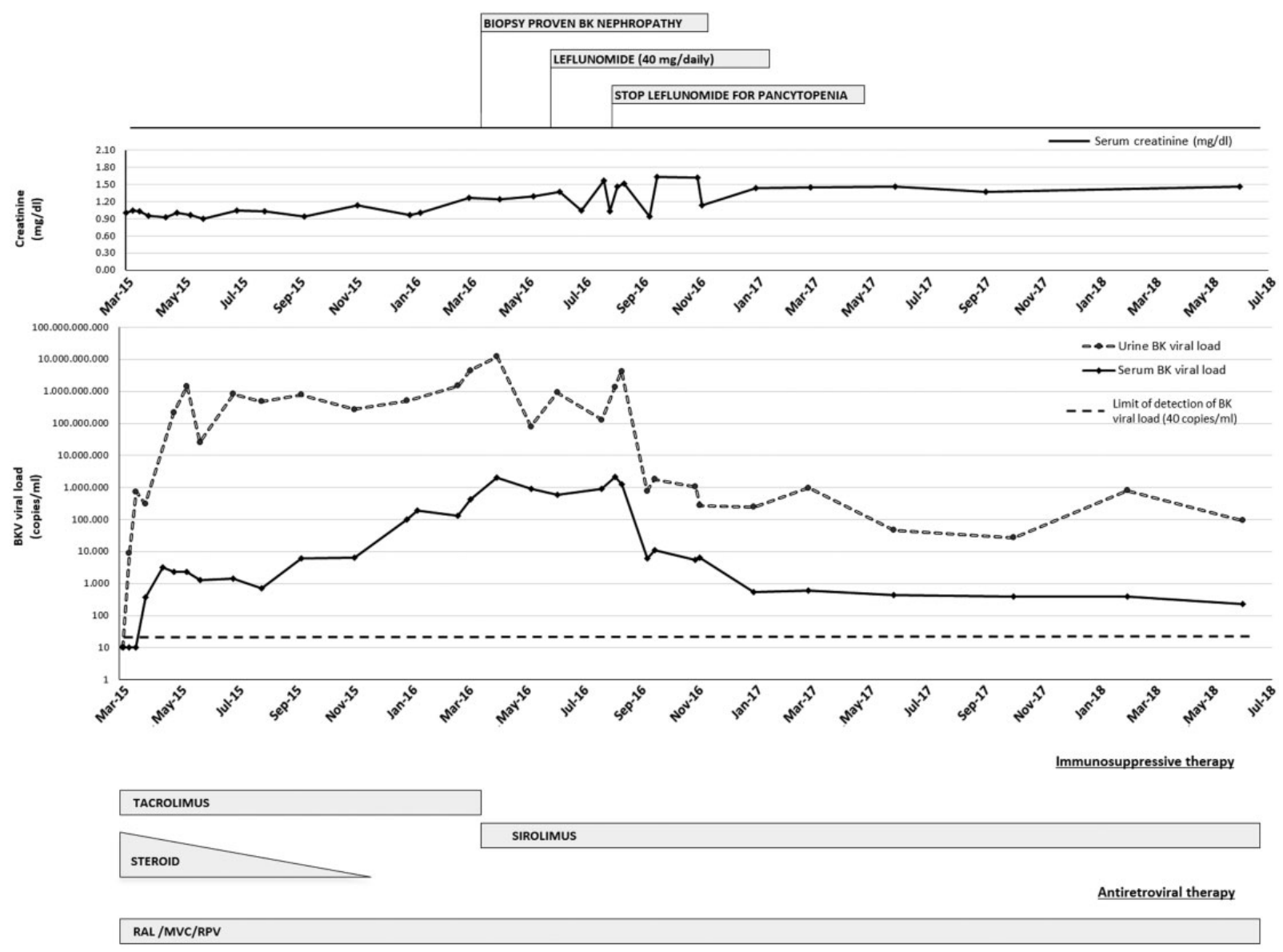

Figure 2. Schematic representation of antiretroviral and immunosuppressive therapies, serum creatinine (mg/dl), and BKV viremia and viruria (copies/ml) after kidney transplantation.

MVC: maraviroc; RAL: raltegravir; RPV: rilpivirine.

$38 \mathrm{ml} / \mathrm{min}$. Urinalysis was negative as was the detection of DSA. After 1.5 years from the introduction of SIR (mean trough level of $7.23 \mathrm{mg} / \mathrm{dl}$ ), no episodes of acute allograft rejection or SIR-associated side effects had occurred in our patient.

\section{Discussion}

With this case report we present our successful experience in controlling BKV replication in an HIV-positive KT recipient with a suppressed HIVVL. BKV infection was responsible for the development of BKVAN and graft impairment in the early post-transplantation period. The infection was managed with a combined strategy including conversion from TAC to SIR, steroid withdrawal and administration of an immunomodulatory drug (leflunomide). We decided to use SIR given its clear antiviral properties against some pathogens (i.e., CMV, HHV-8) commonly encountered after solid organ transplantation. After these therapeutic changes, the profile of BKV replication progressively improved until reaching a clinically insignificant VL and eliminating the risk of graft loss due to the BKV-related tubulointerstitial damage. Given the multiple therapeutic changes, we are unable to separate clearly the beneficial effects of every single intervention on the resolution of $\mathrm{BKV}$ infection. Likely, leflunomide had little or no effect in controlling BKV replication because exposure to this drug was too short to modulate the antiviral response of the host; furthermore, it is still unclear if this drug is really effective in the treatment of BKV infection. ${ }^{15}$

Hence, reduction of immunosuppression with steroid withdrawal, conversion to a weaker immunosuppressive agent with antiproliferative proprieties and likely administration of an immunomodulatory agent with antiviral activity had a pivotal role in the resolution of BKVAN.

SIR, with its immunosuppressive and antiviral activity, is theoretically the most suitable agent to use in KT recipients with BKV infections. Liacini et al. ${ }^{16}$ demonstrated that SIR is able to impair intracellular signaling 
pathways activated by BKV once the virus infects the cells. Recently, two in vivo studies have shown that rapamycin can modulate memory $\mathrm{CD} 8+\mathrm{T}$-cell differentiation in mice. ${ }^{17,18}$ Surprisingly, this immunosuppressive agent improves both quantity and quality of memory CD8 $+\mathrm{T}$ cells stimulated by pathogens. SIR also possesses anti-HIV viral activity. Heredia et al ${ }^{19}$ documented, in an elegant in vitro study, that SIR reduced the expression of CCR5, a co-receptor used by HIV-1 to enter and infect CD4-expressing immune cells. SIR is therefore able to control HIV replication in both HIV-positive liver ${ }^{20}$ and $\mathrm{KT}^{21}$ recipients and to reduce the level of persistent cell-associated HIV DNA in HIV-positive KT recipients. ${ }^{22}$

In our patient, the beneficial antiproliferative effects of SIR were achieved without reaching toxic blood levels. The immunosoppressive agent was well tolerated, and no side effects such as heavy proteinuria, edema, dyslipidemia, oral aphthous ulcers, and pneumonitis developed during the follow-up period. This strategy also enabled the avoidance of graft rejection, a frequent and problematic complication in HIVpositive KT recipients. ${ }^{23}$ However, the use of SIR has to be balanced against an alarming risk of worse longterm graft and patient outcomes compared to the standard immunosuppressive therapy including calcineurin inhibitors. ${ }^{24-26}$ Although we were aware of this risk, we decided to maintain the same immunosuppression because of the persistent low-level replication of BKV despite a suppressed HIV VL and a normal CD4+ Tcell count.

\section{Conclusion}

Reduction of immunosuppression, conversion from calcineurin inhibitors to SIR and, likely, the use of immunomodulatory agent should be taken into consideration in HIV-positive KT recipients with BKV replication, as this infection is a notable cause of graft failure. In this case, conversion to SIR was safe and not associated with renal rejection or drug side-effects.

\section{Declaration of conflicting interests}

The author(s) declared no potential conflicts of interest with respect to the research, authorship, and/or publication of this article.

\section{Funding}

The author(s) received no financial support for the research, authorship, and/or publication of this article.

\section{ORCID iD}

Gaetano Alfano (D) https://orcid.org/0000-0003-0591-8622

\section{References}

1. Gathogo EN, Hamzah L, Hilton R, et al. Kidney transplantation in HIV-positive adults: the UK experience. Int J STD AIDS 2014; 25: 57-66.

2. Locke JE, Gustafson S, Mehta S, et al. Survival benefit of kidney transplantation in HIV-infected patients. Ann Surg 2017; 265: 604-608.

3. Alfano G, Mori G, Fontana F, et al. Clinical outcome of kidney transplantation in HIV-infected recipients: a retrospective study. Int J STD AIDS 2018; 29: 1305-1315.

4. Alfano G, Cappelli G, Fontana F, et al. Kidney disease in HIV infection. J Clin Med 2019; 8: 1254.

5. Sawinski D and Trofe-Clark J. BK virus nephropathy. Clin J Am Soc Nephrol 2018; 13: 1893-1896.

6. Baek $\mathrm{CH}, \mathrm{Kim} \mathrm{H}, \mathrm{Yu} \mathrm{H}$, et al. Risk factors of acute rejection in patients with $\mathrm{BK}$ nephropathy after reduction of immunosuppression. Ann Transplant 2018; 23: 704-712.

7. Cheungpasitporn W, Kremers WK, Lorenz E, et al. De novo donor-specific antibody following BK nephropathy: the incidence and association with antibodymediated rejection. Clin Transplant 2018; 32: e13194.

8. Jacobi J, Prignitz A, Büttner M, et al. BK viremia and polyomavirus nephropathy in 352 kidney transplants; risk factors and potential role of mTOR inhibition. BMC Nephrol 2013; 14: 207.

9. Moscarelli L, Caroti L, Antognoli G, et al. Everolimus leads to a lower risk of BKV viremia than mycophenolic acid in de novo renal transplantation patients: a singlecenter experience. Clin Transplant 2013; 27: 546-554.

10. Schwarz A, Linnenweber-Held S, Heim A, et al. Factors influencing viral clearing and renal function during polyomavirus BK-associated nephropathy after renal transplantation. Transplantation 2012; 94: 396-402.

11. Tohme FA, Kalil RS and Thomas CP. Conversion to a sirolimus-based regimen is associated with lower incidence of BK viremia in low-risk kidney transplant recipients. Transpl Infect Dis off Dis 2015; 17: 66-72.

12. Polanco N, González Monte E, Folgueira MD, et al. Everolimus-based immunosuppression therapy for BK virus nephropathy. Transplant Proc 2015; 47: 57-61.

13. Wojciechowski D, Chandran S, Webber A, et al. Mycophenolate mofetil withdrawal with conversion to everolimus to treat BK virus infection in kidney transplant recipients. Transplant Proc 2017; 49: 1773-1778.

14. Levey AS and Stevens LA. Estimating GFR using the CKD epidemiology collaboration (CKD-EPI) creatinine equation: more accurate GFR estimates, lower CKD prevalence estimates, and better risk predictions. Am J Kidney Dis off Dis 2010; 55: 622-627.

15. Keller N, Duquennoy S, Conrad A, et al. Clinical utility of leflunomide for BK polyomavirus associated nephropathy in kidney transplant recipients: a multicenter retrospective study. Transpl Infect Dis off Dis 2019; 21: e13058.

16. Liacini A, Seamone ME, Muruve DA, et al. Anti-BK virus mechanisms of sirolimus and leflunomide alone and in combination: toward a new 
therapy for BK virus infection. Transplantation 2010; 90: 1450-1457.

17. Pearce EL, Walsh MC, Cejas PJ, et al. Enhancing CD8 T-cell memory by modulating fatty acid metabolism. Nature 2009; 460: 103-107.

18. Araki K, Turner AP, Shaffer VO, et al. mTOR regulates memory CD8 T-cell differentiation. Nature 2009; 460: 108-112.

19. Heredia A, Amoroso A, Davis C, et al. Rapamycin causes down-regulation of CCR5 and accumulation of anti-HIV beta-chemokines: an approach to suppress R5 strains of HIV-1. Proc Natl Acad Sci USA 2003; 100: 10411-10416.

20. Di Benedetto F, Di Sandro S, De Ruvo N, et al. First report on a series of HIV patients undergoing rapamycin monotherapy after liver transplantation. Transplantation 2010; 89: 733-738.

21. Alfano G, Fontana F, Mori G, et al. Antiviral activity of sirolimus in an HIV-positive kidney transplant recipient. Int J STD AIDS 2019; 30: 919-922.
22. Stock PG, Barin B, Hatano H, et al. Reduction of HIV persistence following transplantation in HIV-infected kidney transplant recipients. Am J Transplant 2014; 14: 1136-1141.

23. Sawinski D. Kidney transplantation for HIV-positive patients. Transplant Rev (Orlando) 2017; 31: 42-46.

24. Knoll GA, Kokolo MB, Mallick R, et al. Effect of sirolimus on malignancy and survival after kidney transplantation: systematic review and meta-analysis of individual patient data. BMJ 2014; 349: g6679.

25. Santos AH, Casey MJ, Xuerong W, et al. Association of baseline viral serology and sirolimus regimens with kidney transplant outcomes: a 14-year registry-based cohort study in the United States. Transplantation 2017; 101: 377-386.

26. Isakova $\mathrm{T}$, Xie $\mathrm{H}$, Messinger $\mathrm{S}$, et al. Inhibitors of mTOR and risks of allograft failure and mortality in kidney transplantation. Am J Transplant off Transplant 2013; 13: $100-110$. 